\title{
Experimental realization of two-dimensional synthetic spin-orbit coupling in ultracold Fermi gases
}

\author{
Lianghui Huang ${ }^{1,2}$, Zengming Meng ${ }^{1,2}$, Pengjun Wang ${ }^{1,2}$, Peng Peng ${ }^{1,2}$, Shao-Liang Zhang ${ }^{3}$, \\ Liangchao Chen ${ }^{1}$, Donghao Li', Qi Zhou ${ }^{3 \star}$ and Jing Zhang ${ }^{1,4 \star}$
}

\begin{abstract}
Spin-orbit coupling (SOC) is central to many physical phenomena, including fine structures of atomic spectra and topological phases in ultracold atoms. Whereas, in general, SOC is fixed in a system, laser-atom interaction provides a means to create and control synthetic SOC in ultracold atoms ${ }^{1}$. Despite significant experimental progress in this area $^{2-8}$, two-dimensional (2D) synthetic SOC, which is crucial for exploring two- and threedimensional topological phases, is lacking. Here, we report the experimental realization of 2D SOC in ultracold ${ }^{40} \mathrm{~K}$ Fermi gases using three lasers, each of which dresses one atomic hyperfine spin state. Through spin-injection radiofrequency (rf) spectroscopy ${ }^{4}$, we probe the spin-resolved energy dispersions of the dressed atoms, and observe a highly controllable Dirac point created by the 2D SOC. These results constitute a step towards the realization of new topological states of matter.
\end{abstract}

There have been many theoretical proposals for creating multi-dimensional SOC in ultracold atoms ${ }^{9-14}$, so as to access novel macroscopic quantum phenomena and quantum topological states ${ }^{15-24}$. Whereas these proposals have not been realized in laboratories, physicists have also just begun to explore topological phenomena in optical lattices ${ }^{25-28}$. Here, we use the Raman scheme to produce a highly controllable 2D synthetic SOC for an ultracold Fermi gas of ${ }^{40} \mathrm{~K}$. Such SOC allows us to create and manipulate a single stable Dirac point on a $2 \mathrm{D}$ plane, which is detected by spin-injection rf spectroscopy ${ }^{4}$.

We apply three far-detuned lasers propagating on the $x-y$ plane to couple three ground hyperfine spin states, within the $4^{2} S_{1 / 2}$ ground electronic manifold, $|1\rangle=\left|F=9 / 2, m_{\mathrm{F}}=3 / 2\right\rangle$, $|2\rangle=\left|F=9 / 2, m_{\mathrm{F}}=1 / 2\right\rangle$ and $|3\rangle=\left|F=7 / 2, m_{\mathrm{F}}=1 / 2\right\rangle$, where $\left(F, m_{\mathrm{F}}\right)$ are the quantum numbers for hyperfine spin states as shown in Fig. 1a, to the electronically excited states. Unlike the tripod scheme, where a single excited state is considered ${ }^{9-11,15-18}$, in the ${ }^{40} \mathrm{~K}$ used here the excited states include a fine-structure doublet $4^{2} P_{1 / 2} \quad\left(D_{1}\right.$ line $)$ and $4^{2} P_{3 / 2} \quad\left(D_{2}\right.$ line $)$ with a finestructure splitting of $\sim 3.4 \mathrm{~nm}$. Each of two D-line components also has hyperfine structures. After adiabatically eliminating excited states, the ring scheme proposed in ref. 12 is realized for three cyclically coupled states, with a generalization to arbitrary laser configurations.
The Hamiltonian is written as

$$
\begin{aligned}
H= & \sum_{i=1}^{3}\left(\frac{\mathbf{p}^{2}}{2 m}+\varepsilon_{i}\right)|i\rangle\left\langle i\left|+\sum_{j=1}^{n} E_{j}\right| j\right\rangle\langle j| \\
& +\sum_{i=1}^{3}\left(\Omega_{i} e^{i\left(\mathbf{k}_{i}^{\prime} \cdot \mathbf{r}+\omega_{i} t+\theta_{i}\right)}\left(\sum_{j=1}^{n} M_{j i}|j\rangle\langle i|\right)+\text { h.c. }\right)
\end{aligned}
$$

where $\mathbf{p}$ denotes the momentum of atoms, $\mathbf{k}_{i}^{\prime}\left(\left|\mathbf{k}_{i}^{\prime}\right|=2 \pi / \lambda_{i}\right)$ and $\omega_{i}$ are the wavevectors and frequencies of the three lasers, $\Omega_{i}$ are the Rabi frequencies, $i, j$ are the indices for the three ground hyperfine spin and the excited states respectively, $\varepsilon_{i}$ and $E_{j}$ are the ground and excited state energies, $n$ is the total number of the excited states and $M_{i j}$ is the matrix element of the dipole transition. Different from refs $9,10,15$, each hyperfine ground spin state here is dressed by only one laser field, regardless of the excited states it is coupled to. A gauge transformation, $|i\rangle \rightarrow \mathrm{e}^{-i\left(\mathbf{k}_{i}^{\prime} \cdot \mathbf{r}+\theta_{i}\right)}|i\rangle$, is applied to eliminate the phase $\theta_{i}$. All results discussed here are therefore insensitive to the phase difference, and the sophisticated and challenging phase lockings are no longer necessary.

Applying the rotating wave approximation and the adiabatic elimination of excited states, the Hamiltonian is written as $H_{a}=p_{z}^{2} /(2 m)+H_{x y}$,

$$
H_{x y}=\sum_{i=1}^{3}\left(\frac{\left(\mathbf{p}-\mathbf{k}_{i}\right)^{2}}{2 m}+\delta_{i}\right)|i\rangle\left\langle i\left|-\sum_{i^{\prime} \neq i} \frac{\Omega_{i i^{\prime}}}{2}\right| i\right\rangle\left\langle i^{\prime}\right|
$$

The light-induced energy shifts in the adiabatic elimination have been recast into the diagonal terms, $\delta_{1}$ is set as zero (energy reference) for simplification, $\delta_{2}\left(\delta_{3}\right)$ corresponds to the two-photon Raman detuning between Raman laser 1 and 2 (1 and 3), and $\mathbf{k}_{i}=\hbar \mathbf{k}_{i}^{\prime}$. All three $\Omega_{i i^{\prime}}=\Omega_{i^{\prime} i}$ are real Raman coupling strengths regarding $|i\rangle \leftrightarrow\left|i^{\prime}\right\rangle$. Their values are derived from calculations (see Supplementary Information) and measured in experiments ${ }^{3}$. The single-photon recoil momentum $k_{\mathrm{r}}=2 \pi \hbar / \lambda$ and recoil energy $E_{\mathrm{r}}=k_{\mathrm{r}}^{2} / 2 m$ are taken as natural momentum and energy units. Since the dispersion along the $z$ direction is not affected by the lasers, we will focus on the 2D Hamiltonian $H_{x y}$.

\footnotetext{
${ }^{1}$ State Key Laboratory of Quantum Optics and Quantum Optics Devices, Institute of Opto-Electronics, Shanxi University, Taiyuan 030006, China.

${ }^{2}$ Collaborative Innovation Center of Extreme Optics, Shanxi University, Taiyuan 030006, China. ${ }^{3}$ Department of Physics, The Chinese

University of Hong Kong, Shatin, New Territories, Hong Kong. ${ }^{4}$ Synergetic Innovation Center of Quantum Information and Quantum Physics, University of Science and Technology of China, Hefei, Anhui 230026, China. *e-mail: qizhou@phy.cuhk.edu.hk; jzhang74@sxu.edu.cn, jzhang74@yahoo.com
} 
a

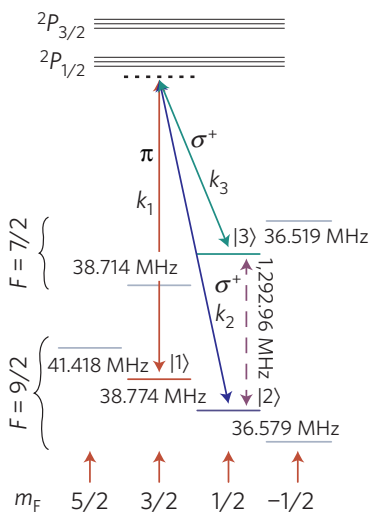

c

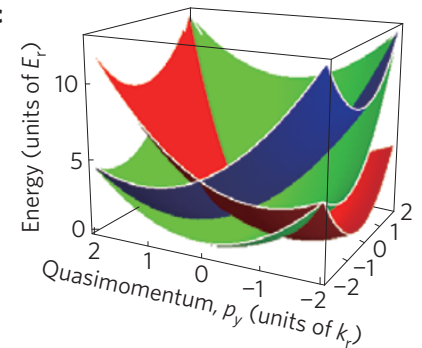

d
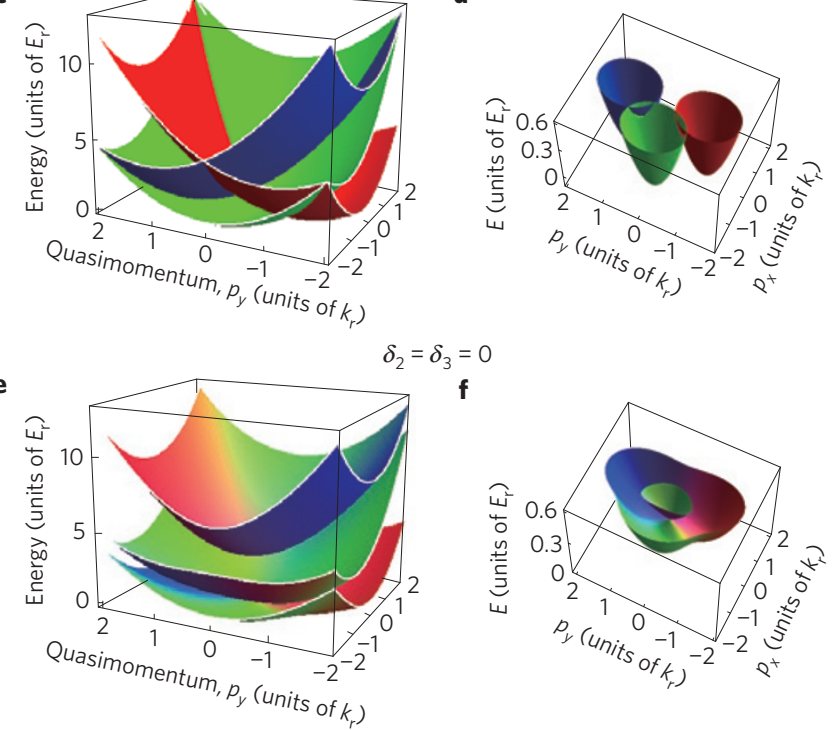

i

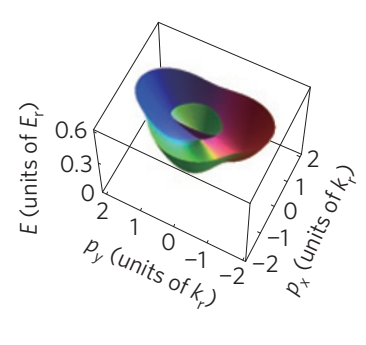

Figure 1 | Two-dimensional synthetic spin-orbit coupling. a, Schematic of energy levels of ${ }^{40} \mathrm{~K}$ for creating 2D SOC. Each of the three Raman lasers dresses one hyperfine spin state from $|9 / 2,3 / 2\rangle(|1\rangle),|9 / 2,1 / 2\rangle(|2\rangle)$ and $|7 / 2,1 / 2\rangle(|3\rangle)$. Atoms are initially prepared in the free reservoir spin state $19 / 2,5 / 2\rangle$. b, Experimental geometry and laser configuration. Raman lasers 1 and 2 counter-propagate along the $y$ axis and Raman laser 3 propagates along the $x$ axis, and they are linearly polarized along the $z, x$ and $y$ directions respectively. c, Energy-momentum dispersions in the absence of SOC $\left(\Omega_{12}=\Omega_{13}=\Omega_{23}=0\right)$. The three parabolae are displaced from the origin by $\mathbf{k}_{i}$, respectively, in the $\left(p_{x}, p_{y}\right)$ plane. $\mathbf{e}$, Energy-momentum dispersions of three dressed states after turning on SOC

( $-\Omega_{12}=2 \Omega_{13}=2 \Omega_{23}=E_{r}$ ), showing that the dispersions of two dressed states touch at a Dirac point. Here, $\delta_{2}=\delta_{3}=0$. d,f, Plots enlarging the low-energy parts of $\mathbf{c}$ and $\mathbf{e}$, respectively. In $\mathbf{e}, \mathbf{f}$, the weights of different hyperfine spin states in the dressed states - the eigenstates of the Hamiltonian with SOC-are represented by assigning different colours to the hyperfine spin states. Red, blue and green represent $|1\rangle,|2\rangle$ and $|3\rangle$ respectively. The depth of each colour indicates the percentage of the corresponding spin state in a dressed state.

Using the two lemmas in Supplementary Information, we find that a doubly degenerate point $\mathbf{p}_{0}$ exists in the momentum space, where $\mathbf{p}_{0}$ satisfies two independent equations

$$
\begin{aligned}
& -\frac{\left(\mathbf{k}_{1}-\mathbf{k}_{2}\right) \cdot \mathbf{p}_{0}}{m}+\delta_{1}-\delta_{2}=-\frac{\Omega_{12} \Omega_{13}}{2 \Omega_{23}}+\frac{\Omega_{12} \Omega_{23}}{2 \Omega_{13}} \\
& -\frac{\left(\mathbf{k}_{2}-\mathbf{k}_{3}\right) \cdot \mathbf{p}_{0}}{m}+\delta_{2}-\delta_{3}=-\frac{\Omega_{12} \Omega_{23}}{2 \Omega_{13}}+\frac{\Omega_{13} \Omega_{23}}{2 \Omega_{12}}
\end{aligned}
$$

Projecting the Hamiltonian in equation (2) to the pseudo-spin$1 / 2$ formed by the two dressed states near the degenerate point $\mathbf{p}_{0}$, an effective Hamiltonian at low energies is obtained,

$$
H_{\mathrm{SO}}=\left(\lambda_{x 1} p_{x}+\lambda_{y 1} p_{y}\right) \sigma_{x}+\left(\lambda_{x 2} p_{x}+\lambda_{y 2} p_{y}\right) \sigma_{z}
$$

where $p_{i=x, y}$ has been redefined as $p_{i}-p_{0, i}$ for simplicity. By rotating the momentum and the spin (see Supplementary Methods), the Hamiltonian can be simplified as

$$
H_{\mathrm{SO}}=\lambda_{x} p_{x}^{\prime} \sigma_{x}^{\prime}+\lambda_{y} p_{y}^{\prime} \sigma_{z}^{\prime}
$$

where both $\lambda_{x}$ and $\lambda_{y}$ are finite. Equation (5) describes a 2D SOC, which is equivalent to the Dresselhaus coupling if a simple transformation $\sigma_{x}^{\prime} \rightarrow \sigma_{y}^{\prime}, \sigma_{z}^{\prime} \rightarrow \sigma_{x}^{\prime}$ is applied. Such a 2D SOC directly tells one that the doubly degenerate point at $\mathbf{p}_{0}$ corresponds to a Dirac point with a linear dispersion at low energies. In particular, both the amplitude and anisotropy $\lambda_{x} / \lambda_{y}$ can be largely tuned (see Supplementary Information). As a demonstration, Figs 1e (1f) and 1c (1d) show a comparison of the energy-momentum dispersion with and without SOC. The latter shows that energy dispersions of two dressed states touch at a Dirac point.

In this experiment, a homogeneous bias magnetic field $B_{0}=121.4 \mathrm{G}$ along the $z$ axis (the gravity direction shown in Fig. 1b) produces a Zeeman shift to isolate these three hyperfine spin states from other ones in the Raman transitions, as shown in Fig. 1a. When the three Raman lasers are nearly resonant with the three ground states $\left(\delta_{i=1,2,3} \approx 0\right)$, the nearest Raman transitions with other hyperfine states are $|7 / 2,3 / 2\rangle \leftrightarrow|2\rangle$ and $|7 / 2,3 / 2\rangle \leftrightarrow|3\rangle$, which have large two-photon Raman detunings about $h \times 60 \mathrm{kHz}$. A three-level system is thus a good description of our experiment.

The Raman coupling strength $\Omega_{i i^{\prime}}$ includes contributions from all excited states of two D-line components, as shown in Fig. 2a (see Supplementary Information). When the wavelength of the Raman lasers is longer than the $\mathrm{D}_{1}$ line $(770.1 \mathrm{~nm})$ or shorter than the $\mathrm{D}_{2}$ line (766.7 nm), $\Omega_{12} \Omega_{13} \Omega_{23}>0$ (see the eigenvalues of dressed states in Supplementary Information), the Dirac point emerges in the highest two dressed states, as shown in Fig. 2b,d. In contrast, when the wavelength of the Raman lasers is tuned between the $D_{1}$ line and $\mathrm{D}_{2}$ line, $\Omega_{12} \Omega_{13} \Omega_{23}<0$, the Dirac point emerges when the lowest two dressed states become degenerate, as shown in Fig. 2c. This is different from the work in ref. 19, where only one excited state is considered and far-blue-detuned lasers are used to make the degenerate dark states the low-lying ones in the manifold of ground electronic states.

We investigate the energy-momentum dispersions of three dressed states by spin-injection rf spectroscopy, which drives the atoms from a free spin-polarized state into SOC dressed states. We start with a degenerate Fermi gas of $2 \times 10^{6}{ }^{40} \mathrm{~K}$ atoms at the free reservoir spin state $|9 / 2,5 / 2\rangle$ in a crossed optical dipole trap. A homogeneous bias magnetic field is ramped to $B_{0}=121.4 \mathrm{G}$. Then the three Raman lasers are ramped up in $60 \mathrm{~ms}$ from zero to their final value. Subsequently, a Gaussian shape pulse of the rf field is applied for $450 \mu$ s to drive atoms from $|9 / 2,5 / 2\rangle$ to the final empty state with $2 \mathrm{D}$ SOC. The rf field does not transfer momentum to the atoms, and spin injection occurs when its frequency matches the energy difference between the initial and final states. Since the spin state $|9 / 2,5 / 2\rangle$ is coupled via rf to the state $|1\rangle$, rf spectroscopy also measures the weight of the $|1\rangle$ state, in addition to the energy dispersions with 2D SOC. Following the spin-injection process, the Raman lasers, the optical trap and the magnetic field are switched off abruptly, and atoms freely expand for $12 \mathrm{~ms}$ in a magnetic field gradient applied along the $x$ axis. Absorption images are taken along the $z$ direction. By counting the number of atoms in state $|1\rangle$ as a function of the momentum and the rf frequency from 
a

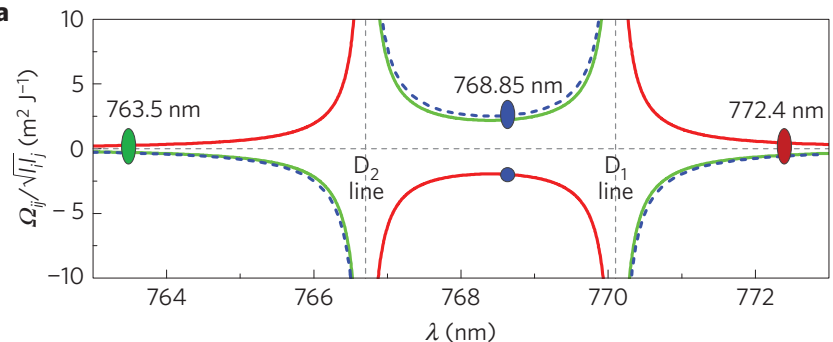

- $|9 / 2,3 / 2\rangle$ and $|9 / 2,1 / 2\rangle \Omega_{12} / \sqrt{I_{1} l_{2}}-|9 / 2,3 / 2\rangle$ and $|7 / 2,1 / 2\rangle \Omega_{13} / \sqrt{I_{1} I_{3}}$ -.. $|9 / 2,1 / 2\rangle$ and $|7 / 2,1 / 2\rangle \Omega_{23} / \sqrt{I_{2} I_{3}}$

b

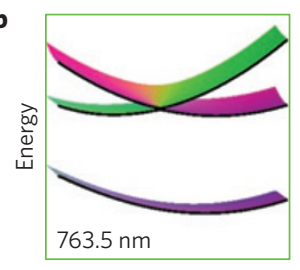

$p_{x}$

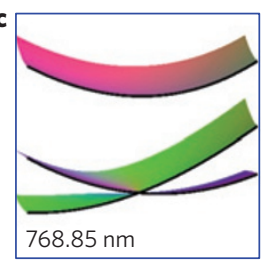

$p_{x}$

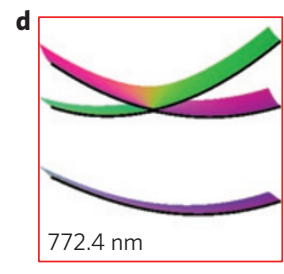

$p_{x}$
Figure 2 | Controlling the Dirac point by changing the wavelength of the Raman lasers. a, Red, green and blue curves represent the Raman coupling strength $\Omega_{12}, \Omega_{13}$ and $\Omega_{23}$ as functions of the wavelength $\lambda$ of the Raman lasers. Depending on $\lambda, \Omega_{12} \Omega_{13} \Omega_{23}$ can be either positive or negative. $l_{i}$ is the intensity of the ith laser. $\mathbf{b}$-d, Energy bands for wavelengths of $763.5 \mathrm{~nm}$ (blue-detuning $D_{2}$ line), $768.85 \mathrm{~nm}$ (between $D_{1}$ and $D_{2}$ line) and $772.4 \mathrm{~nm}$ (red-detuning $D_{1}$ line), respectively. The two higher dispersions touch at the Dirac point in $\mathbf{b}$ and $\mathbf{d}$, where $\Omega_{12} \Omega_{13} \Omega_{23}$ is positive. The two lower dispersions touch at the Dirac point in c, where $\Omega_{12} \Omega_{13} \Omega_{23}$ is negative.

the absorption image, we determine the energy band structure and locate the Dirac point.

Figure $3 \mathrm{a} 2$ shows the momentum-resolved spin-injection spectrum when the one-photon detuning of the Raman lasers is set at a wavelength of $772.4 \mathrm{~nm}$ (red-detuning $\mathrm{D}_{1}$ ); the corresponding theoretical plot is shown in Fig. 3a1. The two higher-energy dispersions touch at a Dirac point, as shown in Fig. 3a2. To further visualize the Dirac point, we plot the energy as a function of $p_{y}$ for various $p_{x}$, as shown in Fig. 3a3-a5. When the wavelength of the Raman lasers is tuned to $768.85 \mathrm{~nm}$, between the $\mathrm{D}_{1}$ line and $\mathrm{D}_{2}$ line, two lower-energy dispersions touch at a Dirac point, as shown in Fig. 3b1-b5. These two wavelengths were used to investigate the 1D SOC (refs 3,5). For the wavelength of the Raman lasers at the $763.5 \mathrm{~nm}$ blue-detuning $\mathrm{D}_{2}$ line, we have observed that two higher-energy dispersions touch at a Dirac point, similar to the $772.4 \mathrm{~nm}$ case. We also perform numerical calculations for the eigenvalues of the Hamiltonian in equations (2) and (5) according to experimental parameters and have found a reasonably good agreement between theory and experiments. In practice, several mechanisms, such as the fluctuation of the magnetic field and the finite momentum resolution, lead to the differences in $\mathrm{rf}$ spectrum between the theoretical and experimental results, as well as the error bars presented in Fig. 3 (see Supplementary Information).

Although a pair of Dirac points has been produced in optical lattices ${ }^{25}$, to our best knowledge, we have created a single Dirac point for the first time in the continuum. Moreover, the Dirac point created in this scheme is robust, in the sense that it moves in momentum space without opening a gap when experimental parameters change. This is because the Hamiltonian in equation (2) is real-consequently, the SOC in equation (5) cannot contain the $\sigma_{y}$ term. By modulating $\delta_{i}, \Omega_{i}$ and $\mathbf{k}_{i}$, an arbitrary trajectory of the Dirac point in the momentum space can in principle be designed. As a demonstration, we fix $\delta_{2}$ and measure the positions of the Dirac point on the $\left(p_{x}, p_{y}\right)$ plane as a function of $\delta_{3}$, as shown a1

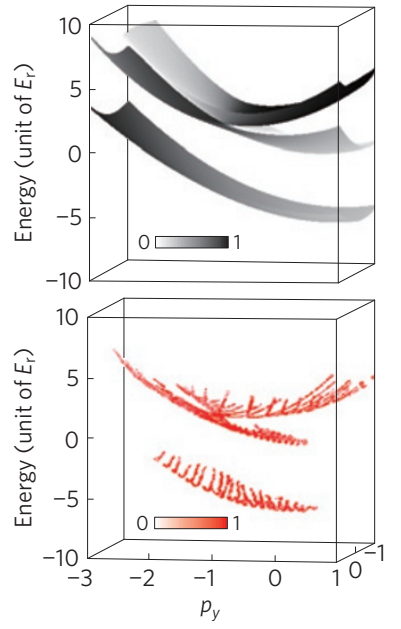

a3

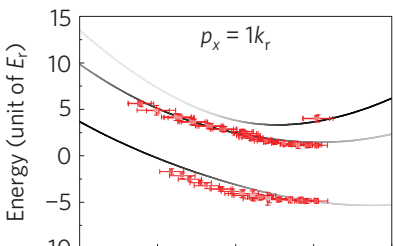

a4

a5

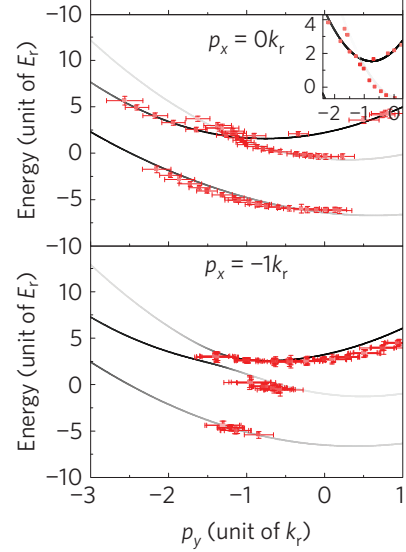

b1

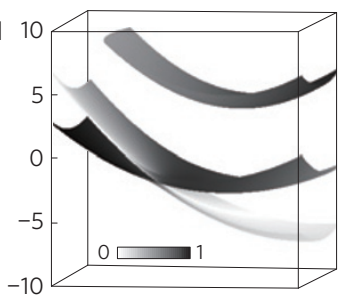

b2

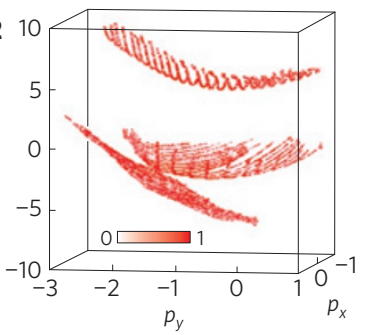

b3

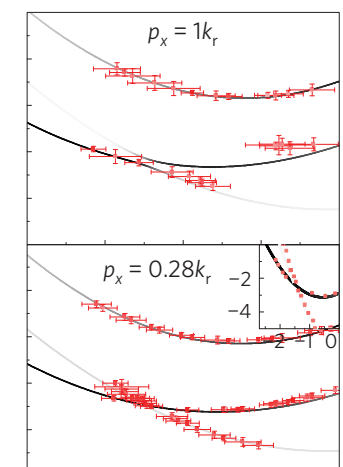

b5

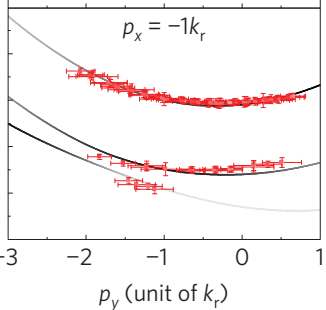

Figure 3 | Energy dispersions of dressed atoms measured by rf spin-injection spectroscopy. a1-a5, The one-photon detuning of the Raman lasers is set at a wavelength of $772.4 \mathrm{~nm}$ (red-detuning $\left.D_{1}\right) . \Omega_{12}=3.58 E_{r_{1}}$, $\Omega_{13}=-3.94 E_{r}, \Omega_{23}=-4.66 E_{r}, \delta_{2}=-5.14 E_{r}, \delta_{3}=-3.23 E_{r}$. b1-b5, The wavelength is set at $768.85 \mathrm{~nm}$, between the $D_{1}$ line and $D_{2}$ line. $\Omega_{12}=-4.96 E_{r}, \Omega_{13}=5.46 E_{r}, \Omega_{23}=6.46 E_{r}, \delta_{2}=-5.2 E_{r}, \delta_{3}=-2.13 E_{r}$. a1,b1, Theoretical results for the $|1\rangle$ component using the realistic experimental parameters. $\mathbf{a 2}, \mathbf{b 2}$, Experimental results measured by rf spin-injection spectroscopy. Each line on the surfaces of the dispersions is constructed from time-of-flight images at the given rf frequency (see Supplementary Information). a3-a5, Cross-section drawings of $\mathbf{a} \mathbf{1}$ and $\mathbf{a} \mathbf{2}$ in energy- $p_{y}$ coordinates for different quasimomenta $p_{x}$. Red dots with error bars and solid curves are experimental and theoretical results, respectively. The inset in $\mathbf{a} \mathbf{4}$ is a plot (without error bars) enlarging the region near the Dirac point. Here, $\lambda_{x 1}=0, \lambda_{x 2}=-0.42 k_{r} / m, \lambda_{y 1}=1.5 k_{r} / m$, and $\lambda_{y 2}=-0.07 k_{r} / m$ are obtained by the experimental parameters. b3-b5, Cross-section drawings of $\mathbf{b} \mathbf{1}$ and $\mathbf{b} \mathbf{2}$. The inset in $\mathbf{b} \mathbf{4}$ is a plot enlarging the region near the Dirac point. $\lambda_{x 1}, \lambda_{x 2} \lambda_{y 1}$ and $\lambda_{y 2}$ are the same as in $\mathbf{a}$, since $\Omega_{12} / \Omega_{13}$ and $\Omega_{12} / \Omega_{23}$ remain the same.

in Fig. 4a (blue dots). When fixing $\delta_{3}$ and changing $\delta_{2}$, the Dirac point moves along a different line (red dots). We also measure the energy at the Dirac point, as well as the energy separation $\Delta E$ from highest dressed state, as a function of the Raman coupling strength. Zero energy in Fig. $4 \mathrm{~b}$ corresponds to the Zeeman energy splitting between $|9 / 2,5 / 2\rangle$ and $|9 / 2,3 / 2\rangle$. The energy of the Dirac point 
a

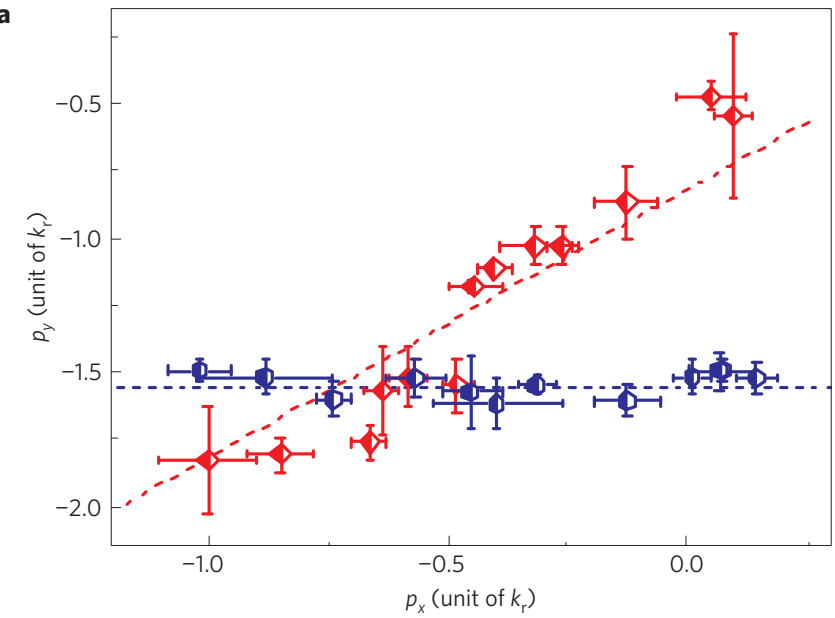

b

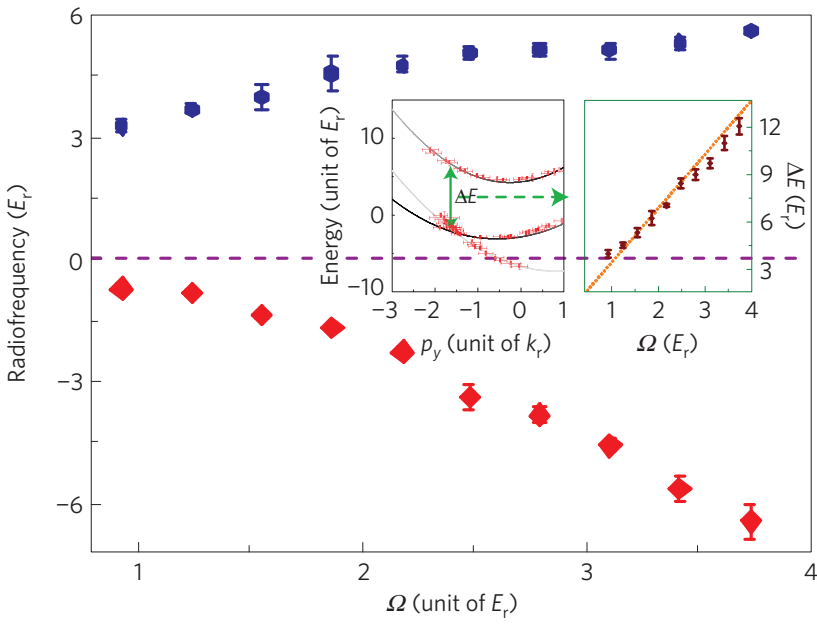

Figure 4 | Moving the Dirac point. a, Controlling the Raman detuning $\delta_{2}$ and $\delta_{3}$, the Dirac point moves along certain trajectories in momentum space. Blue dots: $\delta_{2}$ is fixed at $-5.45 E_{\mathrm{r}}$ and $\delta_{3}$ is tuned in the range $\left[-2 E_{r}, 0.45 E_{r}\right]$. Red dots: $\delta_{3}$ is fixed at $-0.2 E_{r}$ and $\delta_{2}$ is tuned in the range $\left[-7 E_{r},-2.5 E_{r}\right]$. The other parameters are $\Omega_{12}=-4.96 E_{r}, \Omega_{13}=5.46 E_{r}$, $\Omega_{23}=6.46 E_{\mathrm{r}}$. b, Energy at the Dirac point (red diamonds) and corresponding energy of the highest dressed state with the same momentum $\mathbf{p}_{0}$ (blue boxes) as a function of the Raman coupling strength. $-\Omega_{12}=0.91 \Omega_{13}=0.77 \Omega_{23}=\Omega, \delta_{2}=-5.2 E_{\mathrm{r}}$ and $\delta_{3}=-2.13 E_{\mathrm{r}}$. The left inset in $\mathbf{b}$ shows the energy dispersions. The right inset in $\mathbf{b}$ represents the energy separation $\Delta E$ between the Dirac point and the highest dressed state as a function of the Raman coupling strength. Here, the one-photon detuning of three Raman lasers is at a wavelength of $768.85 \mathrm{~nm}$. The error bars represent the standard deviation of repeated measurements.

decreases with increasing Raman coupling strength (red diamonds). $\Delta E$ increases linearly with increasing Raman coupling strength (right inset of Fig. 4b). Since the diagonal energy shift induced by the Raman field has no influence on the effective SOC, we further plot the energy difference between the Dirac point and the highest dressed state, as highlighted by the green vertical arrow in the left inset of Fig. 4b. We find that $\Delta E$ increases linearly with increasing Raman coupling strength (right inset of Fig. 4b), and observe a good agreement between experimental and theoretical results.

We hope that a highly controllable 2D SOC will help physicists to explore both gapless topological matter in the continuum, analogous to graphene and Weyl semimetals in lattices, and interesting topological or other exotic superfluids when the gap at the Dirac point is opened ${ }^{20-24}$. Whereas our scheme is very general and can be applied to any atomic species, we hope that the specific heating effect in alkali atoms due to the fine-structure splitting may be overcome by novel cooling schemes or the quantum quenching approach, which may allow one to extract topological properties from quantum dynamics in a relatively short timescale ${ }^{29}$.

\section{Methods}

Methods and any associated references are available in the online version of the paper.

Received 25 October 2015; accepted 21 January 2016; published online 29 February 2016

\section{References}

1. Dalibard, J., Gerbier, F., Juzeliūnas, G. \& Öhberg, P. Artificial gauge potentials for neutral atoms. Rev. Mod. Phys. 83, 1523-1543 (2011).

2. Lin, Y.-J., Jiménez-García, K. \& Spielman, I. B. Spin-orbit-coupled Bose-Einstein condensates. Nature 471, 83-86 (2011).

3. Wang, P. et al. Spin-orbit coupled degenerate Fermi gases. Phys. Rev. Lett. 109, 095301 (2012)

4. Cheuk, L. W. et al. Spin-injection spectroscopy of a spin-orbit coupled Fermi gas. Phys. Rev. Lett. 109, 095302 (2012).

5. Williams, R. A., Beeler, M. C., LeBlanc, L. J. \& Spielman, I. B. Raman-induced interactions in a single-component Fermi gas near an s-wave Feshbach resonance. Phys. Rev. Lett. 111, 095301 (2013).

6. Zhang, J.-Y. et al. Collective dipole oscillations of a spin-orbit coupled Bose-Einstein condensate. Phys. Rev. Lett. 109, 115301 (2012).

7. Qu, C., Hamner, C., Gong, M., Zhang, C. \& Engels, P. Observation of Zitterbewegung in a spin-orbit coupled Bose-Einstein condensates. Phys. Rev. A 88, 021604(R) (2013).

8. Olson, A. J. et al. Tunable Laudau-Zener transitions in a spin-orbit-coupled Bose-Einstein condensate. Phys. Rev. A 90, 013616 (2014).

9. Unanyan, R. G., Shore, B. W. \& Bergmann, K. Laser-driven population transfer in four-level atoms: consequences of non-Abelian geometrical adiabatic phase factors. Phys. Rev. A 59, 2910-2919 (1999).

10. Ruseckas, J., Juzeliūnas, G., Öhberg, P. \& Fleischhauer, M. Non-Abelian gauge potentials for ultracold atoms with degenerate dark states. Phys. Rev. Lett. 95, 010404 (2005)

11. Juzeliūnas, G., Ruseckas, J. \& Dalibard, J. Generalized Rashba-Dresselhaus spin-orbit coupling for cold atoms. Phys. Rev. A 81, 053403 (2010).

12. Campbell, D. L., Juzeliūnas, G. \& Spielman, I. B. Realistic Rashba and Dresselhaus spin-orbit coupling for neutral atoms. Phys. Rev. A 84, $025602(2011)$

13. Xu, Z. F., You, L. \& Ueda, M. Atomic spin-orbit coupling synthesized with magnetic-field-gradient pulses. Phys. Rev. A 87, 063634 (2013).

14. Anderson, B. M., Spielman, I. B. \& Juzeliūnas, G. Magnetically generated spin-orbit coupling for ultracold atoms. Phys. Rev. Lett. 111, 125301 (2013).

15. Stanescu, T. D., Zhang, C. \& Galitski, V. Nonequilibrium spin dynamics in a trapped Fermi gas with effective spin-orbit interactions. Phys. Rev. Lett. 99, 110403 (2007).

16. Juzeliūnas, G., Ruseckas, J., Jacob, A., Santos, L. \& Öhberg, P. Double and negative reflection of cold atoms in non-Abelian gauge potentials. Phys. Rev. Lett. 100, 200405 (2008).

17. Vaishnav, J. Y. \& Clark, C. W. Observing Zitterbewegung with ultracold atoms. Phys. Rev. Lett. 100, 153002 (2008).

18. Larson, J. \& Sjöqvist, E. Jahn-Teller-induced Berry phase in spin-orbit-coupled Bose-Einstein condensates. Phys. Rev. A 79, 043627 (2009).

19. Zhang, Y., Mao, L. \& Zhang, C. Mean-field dynamics of spin-orbit coupled Bose-Einstein condensates. Phys. Rev. Lett. 108, 035302 (2012).

20. Zhang, C. Spin-orbit coupling and perpendicular Zeeman field for fermionic cold atoms: observation of the intrinsic anomalous Hall effect. Phys. Rev. A 82, 021607(R) (2010)

21. Zhu, S.-L., Shao, L.-B., Wang, Z. D. \& Duan, L.-M. Probing non-Abelian statistics of Majorana fermions in ultracold atomic superfluid. Phys. Rev. Lett. 106, 100404 (2011).

22. Vyasanakere, J. P. \& Shenoy, V. B. Bound states of two spin- $\frac{1}{2}$ fermions in a synthetic non-Abelian gauge field. Phys. Rev. B 83, 094515 (2011).

23. Hu, H., Jiang, L., Liu, X.-J. \& Han, P. Probing anisotropic superfluidity in atomic Fermi gases with Rashba spin-orbit coupling. Phys. Rev. Lett. 107, $195304(2011)$

24. Yu, Z.-Q. \& Zhai, H. Spin-orbit coupled Fermi gases across a Feshbach resonance. Phys. Rev. Lett. 107, 195305 (2011).

25. Tarruell, L., Greif, D., Uehlinger, T., Jotzu, G. \& Esslinger, T. Creating, moving and merging Dirac points with a Fermi gas in a tunable honeycomb lattice. Nature 483, 302-305 (2012) 
26. Jotzu, G. et al. Experimental realization of the topological Haldane model with ultracold fermions. Nature 515, 237-240 (2014).

27. Aidelsburger, M. et al. Realization of the Hofstadter Hamiltonian with ultracold atoms in optical lattices. Phys. Rev. Lett. 111, 185301 (2013).

28. Miyake, H., Siviloglou, G. A., Kennedy, C. J., Burton, W. C. \& Ketterle, W. Realizing the Harper Hamiltonian with laser-assisted tunneling in optical lattices. Phys. Rev. Lett. 111, 185302 (2013).

29. Setiawan, F., Sengupta, K., Spielman, I. B. \& Sau, J. D. Dynamical detection of topological phase transition in short-lived atomic systems. Phys. Rev. Lett. 115, 190401 (2015).

\section{Acknowledgements}

We would like to thank H. Zhai, S. Zhang, C. Zhang, H. Pu, H. Hu, G. Juzeliūnas and B. Anderson for helpful discussions. This research is supported by the National Basic Research Program of China (Grant No. 2011CB921601), NSFC (Grant No. 11234008
$11361161002,11222430)$ and the Program for Sanjin Scholars of Shanxi Province. Q.Z. is supported by NSFC/RGC(NCUHK453/13).

\section{Author contributions}

L.H., Z.M., P.W., P.P., L.C., D.L. and J.Z. performed experiments. S.-L.Z., Q.Z. and J.Z. did the theory. S.-L.Z., Q.Z., L.H., P.W. and J.Z. contributed to the theoretical modelling and explanation of the experimental data. Q.Z. and J.Z. wrote the paper. All authors discussed the results and commented on the manuscript. J.Z. supervised the project.

\section{Additional information}

Supplementary information is available in the online version of the paper. Reprints and permissions information is available online at www.nature.com/reprints.

Correspondence and requests for materials should be addressed to Q.Z. or J.Z.

\section{Competing financial interests}

The authors declare no competing financial interests. 


\section{Methods}

Experimental set-up. After sympathetic cooling with bosonic ${ }^{87} \mathrm{Rb}$ in a quadrupole-Ioffe configuration magnetic trap, ${ }^{40} \mathrm{~K}$ atoms in the $|9 / 2,9 / 2\rangle$ state and ${ }^{87} \mathrm{Rb}$ atoms in the $|2,2\rangle$ are transferred into an optical dipole trap formed by two orthogonal 1,064 nm laser beams. The Fermi gas is further evaporatively cooled to $T / T_{\mathrm{F}} \approx 0.3$, giving about $2 \times 10^{640} \mathrm{~K}$ atoms with ${ }^{87} \mathrm{Rb}$ atoms in the optical trap ${ }^{30}$, where $T_{\mathrm{F}}$ is the Fermi temperature defined by $T_{\mathrm{F}}=(6 N)^{1 / 3} \hbar \bar{\omega} / k_{\mathrm{B}}$ and the geometric mean of trapping frequencies $\bar{\omega} \simeq 2 \pi \times 80 \mathrm{~Hz}$ in our system, $N$ is the number of fermions. After removal of the ${ }^{87} \mathrm{Rb}$ atoms, the fermionic atoms are transferred into the state $|9 / 2,5 / 2\rangle$ via a rapid adiabatic passage induced by a rf field of $80 \mathrm{~ms}$ at $19.6 \mathrm{G}$. Here, the transition is addressed by a rf ramp that starts at $6.56 \mathrm{MHz}$ to the end of $6.28 \mathrm{MHz}$. Then a homogeneous bias magnetic field along the $z$ axis (gravity direction) is ramped to $B_{0}=121.4 \mathrm{G}$ by a pair of coils operating in the Helmholtz configuration.

Raman lasers. Three Raman lasers are derived from a CW Ti-sapphire single frequency laser. Two Raman beams 1 and 2 are frequency-shifted around
$+201.144 \times 2 \mathrm{MHz}$ and $+220.531 \times 2 \mathrm{MHz}$ by two double-pass acousto-optic modulators (AOM), respectively. Raman laser 3 is sent through two AOMs in double-pass and frequency-shifted $-212.975 \times 4 \mathrm{MHz}$. Then the three Raman beams are coupled into three polarization-maintaining single-mode fibres, respectively, in order to improve stability of the beam pointing and achieve a better beam-profile quality. After the fibres, these three Raman beams have maximum powers of $80 \mathrm{~mW}$ for each beam and overlap in the atomic cloud with $1 / e^{2}$ radii of $200 \mu \mathrm{m}$. Raman lasers 1 and 2 counter-propagate along the $y$ axis and Raman laser 3 propagates along the $x$ axis, and they are linearly polarized along the $z, x$ and $y$ directions, respectively, corresponding to $\pi, \sigma$ and $\sigma$ polarizations relative to the quantization axis $z$ shown in Fig. 1b.

\section{References}

30. Xiong, D. et al. Quantum degenerate Fermi-Bose mixtures of ${ }^{40} \mathrm{~K}$ and ${ }^{87} \mathrm{Rb}$ atoms in a quadrupole-Ioffe configuration trap. Chin. Phys. Lett. 25, 843-846 (2008) 